\title{
Remote Ischaemic Conditioning in STEMI Patients in Sub-Saharan AFRICA: Rationale and Study Design for the RIC-AFRICA Trial
}

\author{
Kishal Lukhna ${ }^{1}$ - Derek J. Hausenloy ${ }^{2,3,4,5,6}$. Abdelbagi Sidahmed Ali $^{7} \cdot$ Abdullah Bajaber $^{8}$ - Alistair Calver ${ }^{9}$. \\ Arthur Mutyaba $^{10}$. Awad Abdalla Mohamed ${ }^{11,12} \cdot$ Brian Kiggundu $^{13} \cdot$ Chishala Chishala $^{14} \cdot$ Ebrahim Variava $^{9}$. \\ Ehab Ali Elmakki ${ }^{15}$. Elijah Ogola ${ }^{16}$. Eltayeb Hamid ${ }^{11}$. Emmy Okello ${ }^{13}$. Isam Gaafar ${ }^{17} \cdot$ Keiran Mwazo $^{18}$. \\ Makoali Makotoko ${ }^{19}$. Mergan Naidoo ${ }^{20}$. Mohamed Elhadi Abdelhameed ${ }^{21} \cdot$ Motasim Badri $^{22}$. \\ Nasief van der Schyff ${ }^{23} \cdot$ Omaima Abozaid $^{24} \cdot$ Paul Xafis $^{23} \cdot$ Sara Giesz $^{2} \cdot$ Trevor Gould $^{25} \cdot$ Waldo Welgemoed $^{25}$. \\ Malcolm Walker ${ }^{2} \cdot$ Mpiko Ntsekhe $^{1} \cdot$ Derek M Yellon $^{2}{ }^{\circ}$
}

Accepted: 25 October 2021 / Published online: 5 November 2021

(c) The Author(s) 2021

\begin{abstract}
Purpose Despite evidence of myocardial infarct size reduction in animal studies, remote ischaemic conditioning (RIC) failed to improve clinical outcomes in the large CONDI-2/ERIC-PPCI trial. Potential reasons include that the predominantly low-risk study participants all received timely optimal reperfusion therapy by primary percutaneous coronary intervention (PPCI). Whether RIC can improve clinical outcomes in higher-risk STEMI patients in environments with poor access to early reperfusion or PPCI will be investigated in the RIC-AFRICA trial.

Methods The RIC-AFRICA study is a sub-Saharan African multi-centre, randomized, double-blind, sham-controlled clinical trial designed to test the impact of RIC on the composite endpoint of 30-day mortality and heart failure in 1200 adult STEMI patients without access to PPCI. Randomized participants will be stratified by whether or not they receive thrombolytic therapy within $12 \mathrm{~h}$ or arrive outside the thrombolytic window (12-24 h). Participants will receive either RIC (four 5-min cycles of inflation [ $20 \mathrm{mmHg}$ above systolic blood pressure] and deflation of an automated blood pressure cuff placed on the upper arm) or sham control (similar protocol but with low-pressure inflation of $20 \mathrm{mmHg}$ and deflation) within $1 \mathrm{~h}$ of thrombolysis and applied daily for the next 2 days. STEMI patients arriving greater than $24 \mathrm{~h}$ after chest pain but within 72 $\mathrm{h}$ will be recruited to participate in a concurrently running independent observational arm.

Conclusion The RIC-AFRICA trial will determine whether RIC can reduce rates of death and heart failure in higher-risk sub-optimally reperfused STEMI patients, thereby providing a low-cost, non-invasive therapy for improving health outcomes.
\end{abstract}

Keywords ST elevation myocardial infarction · Cardioprotection · Remote ischaemic conditioning Ischaemia/reperfusion injury $\cdot$ Hospitalization for heart failure

\section{Abbreviations}

AMI Acute myocardial infarction

HF Heart failure

MACCE Major adverse cardiac and cerebrovascular events

MI Myocardial infarction

PCI Percutaneous coronary intervention
PPCI Primary percutaneous coronary intervention

RCT Randomized control trial

RIC Remote ischaemic conditioning

SSA Sub-Saharan Africa

STEMI ST elevation myocardial infarction

cTn Troponin

\section{Introduction}

Kishal Lukhna and Derek J. Hausenloy are joint first authors, and Mpiko Ntsekhe and Derek M Yellon are joint senior authors.

Derek M Yellon

d.yellon@ucl.ac.uk

Extended author information available on the last page of the article
Acute myocardial infarction (AMI) and the heart failure (HF) that often follows are among the leading causes of death and disability in sub-Saharan Africa (SSA) and worldwide [1]. The prevalence of ischaemic heart disease and its related mortality 
rate is predicted to rise by $70 \%$ in African men and $74 \%$ in women by 2030 , and the burden of acute coronary syndromes in sub-Saharan Africa are steadily rising [2, 3]. Observational studies in sub-Saharan Africa have reported high in-patient mortality rates in STEMI patients ranging from 15 to $21 \%$, with unacceptably high post-myocardial infarction (MI) heart failure rates $[1,2]$.

In most high-income countries, patients presenting with an acute ST-segment elevation myocardial infarction (STEMI) undergo immediate revascularization by primary percutaneous coronary intervention (PPCI) as the treatment of choice. However, in many low-and middle-income countries in subSaharan Africa, the availability and access to modern, expensive infrastructure and interventions such as PPCI are limited, and this is unlikely to change in the near future. As a result, of the STEMI patients who receive reperfusion, the majority are treated by thrombolysis and are more likely to develop heart failure and death post-STEMI, highlighting a higher-risk patient population. As such, there remains an urgent unmet need to discover novel therapeutic interventions that will improve clinical outcomes and prevent the onset of heart failure following STEMI.

In this regard, remote ischaemic conditioning (RIC), an endogenous cardioprotective phenomenon in which brief cycles of sublethal ischaemia and reperfusion applied to the limb, has been shown to reduce MI size in small and large animal models of acute myocardial ischaemia/reperfusion injury [4]. Crucially, the cardioprotective stimulus of RIC can be induced by simply inflating and deflating a pneumatic cuff placed on the upper arm or thigh for four 5-min cycles as adjunctive therapy in STEMI patients, making RIC an attractive low-cost and non-invasive treatment strategy for improving clinical outcomes in STEMI patients [5-10]. Although several clinical studies of STEMI patients reported reduced MI size with RIC, the large European CONDI-2/ERICPPCI multi-centre, randomized, clinical trial did not find any improvement in clinical outcomes [11]. Potential reasons for this failure to translate RIC into patient benefit included the low-risk population recruited into that study and the fact that patients who had received optimal reperfusion therapy by PPCI contributed to an unexpectedly low event rate in the sham treatment group, resulting in an unpowered trial [12-14]. In the RIC-AFRICA trial, we investigate whether RIC can improve clinical outcomes in higher-risk STEMI patients in sub-Saharan Africa treated by thrombolysis.

\section{Methods}

\section{Study Design}

The RIC-AFRICA study is a sub-Saharan African prospective, multi-centre, randomized, phase 3 double-blinded, sham-controlled clinical trial of 1200 STEMI patients across 20 sites in South Africa, Kenya, Sudan, and Uganda. Patients who are ineligible because they present after $24 \mathrm{~h}$ will be concurrently recruited into an independent observational arm of the study if they are within $72 \mathrm{~h}$ of MI onset. The study will be conducted in accordance with the Declaration of Helsinki and has received approval from the ethics committee of the University of Cape Town (September 10, 2021/ HREC107.2021). Trial enrolment will begin from December 01, 2021, and extend over a 24-month period. The RIC-AFRICA study protocol has been registered at www. clinicaltrials.gov (identifier: NCT04813159), and the study will be conducted and reported according to the CONSORT statement [15]. An independent data and safety monitoring board has been selected to oversee the trial. All participants will provide informed written consent.

\section{Study Population}

We will be recruiting 3 different strata of STEMI patients.

1. Adult patients ( $\geq 18$ years old) presenting with STEMI receiving thrombolytic therapy within guideline-recommended time (i.e., within \pm 12 hours of most severe chest pain)

2. Adult patients ( $\geq 18$ years old) presenting with STEMI who are ineligible for thrombolysis because they present outside of guideline-recommended time $( \pm 12 \mathrm{~h})$ but within $24 \mathrm{~h}$ of most severe chest pain

3. Adult patients ( $\geq 18$ years old) presenting with evidence of STEMI who do not receive thrombolysis and who present $\geq 24 \mathrm{~h}$ and within $72 \mathrm{~h}$ of most severe chest pain

\section{Interventional Arm: Randomized Control Trial}

Patients who are deemed eligible for randomization into the trial on account of presentation with STEMI within $24 \mathrm{~h}$ will be eligible for the interventional arm of the study if the following inclusion/exclusion criteria are met.

\section{Inclusion Criteria}

I. Adult patients ( $\geq 18$ years old) presenting with suspected STEMI (new ST-elevation at the J-point in two contiguous leads with the cut-points: $\geq 0.1 \mathrm{mV}$ in all leads other than V2-3 where the following cutpoints apply: $\geq 0.2 \mathrm{mV}$ in men $\geq 40$ years $(\geq 0.25 \mathrm{mV}$ in men $<40$ years) or $\geq 0.15 \mathrm{mV}$ in women)

II. Within $24 \mathrm{~h}$ of onset of myocardial infarction as deemed by the attending clinician

III. Signed informed consent 


\section{Exclusion Criteria}

I. STEMI patients undergoing primary percutaneous coronary intervention

II. STEMI patients presenting with cardiogenic shock or haemodynamic instability (systolic blood pressure (SBP) $<90 \mathrm{mmHg}$ for $\geq 30 \mathrm{~min}$, or use of pharmacological and/or mechanical support to maintain SBP $\geq$ $90 \mathrm{mmHg}$, and evidence of end-organ damage such as urine output of $<30 \mathrm{~mL} / \mathrm{h}$, altered mental status, and/or serum lactate $>2.0 \mathrm{mmol} / \mathrm{L}$ ) [16]

III. Contra-indication to thrombolytic therapy in patients presenting within guideline-recommended time $( \pm 12$ h)

IV. Conditions that preclude the use of RIC or shamcontrol on either arm such as:

a. Severe active skin disease/burns on both arms

b. Bilateral upper limb amputations

c. Evidence of acute limb ischaemia on either arm

d. Active upper limb gangrene of any digits

e. Bilateral arteriovenous fistulae needed for haemodialysis

f. Known breast cancer with ipsilateral lymph node involvement on the side of RIC

V. Inter-current disease with an expected life expectancy of less than $24 \mathrm{~h}$

\section{Observational Arm}

There is currently very limited data surrounding late STEMI presentation, management, and outcomes across Africa where the condition is thought to be common. As this will be one of the first large scale interventional STEMI trials conducted across Africa, we have decided to use this opportunity to gather as much information as possible about patients who present with late STEMI to help narrow the gap on this subject in the medical literature, while concurrently generating future research opportunities in Africa. The participants in the observational arm will not be compared to the participants randomized to the trial intervention.

Patients who are deemed ineligible for randomization into the clinical trial on account of presentation beyond $24 \mathrm{~h}$ will be eligible for the observational arm of the study if the following inclusion/exclusion criteria are met.

\section{Inclusion Criteria}

I. Signed informed consent

II. Adult patients ( $\geq 18$ years old) with clinical evidence of STEMI older than $24 \mathrm{~h}$ and less than $72 \mathrm{~h}$ as defined by the following:
a. Compatible history with maximal chest pain between 24 and $72 \mathrm{~h}$ prior to presentation
b. Compatible biomarkers (elevated cardiac troponin)
c. ECG compatible with recent STEMI; and/or
d. Compatible echocardiography

\section{Exclusion Criteria}

I. Refusal or inability to sign informed consent

\section{Randomization}

Consenting participants who present within $24 \mathrm{~h}$ of MI onset will be randomized to receive either RIC or sham control. Randomization will be conducted via an automated, secure web-based program built into the data capturing software. Randomization will be stratified according to recruiting site and time of MI-onset, using random permuted blocks. The randomization schedule will be conducted in a manner that ensures that 2 participants in stratum 1 (receives thrombolysis within $\pm 12 \mathrm{~h}$ of MI onset) are recruited for every participant in stratum 2 (outside the thrombolytic window but within 24 $h$ of MI onset) and will ensure equal allocation to both the active and sham control arms. The patient, treating clinician, and study investigators will be blinded to the treatment allocation. Willing participants who present between 24 and 72 $\mathrm{h}$ of MI onset will be recruited into the concurrently running independent observational arm of the RIC-AFRICA study.

\section{Trial Intervention}

An automated pneumatic blood pressure device (manufactured by Seagull Aps in Denmark) will be used to deliver either the RIC or sham protocol. The RIC protocol will comprise of applying the blood pressure cuff at recruitment on the upper arm to automatically deliver four 5-min alternating high-pressure cuff inflations $(20 \mathrm{mmHg}$ above each participant's systolic blood pressure) and cuff deflations, such that the total RIC protocol takes $40 \mathrm{~min}$. The sham control protocol will comprise the application of a visually identical pneumatic cuff on the upper arm which will automatically deliver four 5-min alternating low-pressure cuff inflations $(20 \mathrm{mmHg}$ ) and cuff deflations, for a total of $40 \mathrm{~min}$. The RIC and sham protocol will be repeated daily over the next two days. Enrolled participants in stratum 1 will receive RIC or sham control within $1 \mathrm{~h}$ of thrombolysis. Participants in stratum 2 will receive RIC or sham control within $1 \mathrm{~h}$ of randomization. The RIC and sham devices will be applied by the unblinded research nurse. None of the study interventions or procedures will interfere with standard STEMI management at each site. Participants enrolled in the observational arm will not receive any trial intervention. 


\section{Study Endpoints}

\section{Primary Endpoint}

For the purpose of this trial, all endpoints were consistent with the 2017 cardiovascular and stroke endpoint definitions for clinical trials consensus document [17].

The primary composite endpoints of the study will be all-cause death and early post-MI heart failure. The latter is defined as (a) pre-discharge (in-hospital) heart failure or (b) heart failure hospitalization within 30 days of discharge from index myocardial infarction (see Table 1 of Appendix for more details).

\section{Secondary Endpoint}

Secondary outcome measures will include a composite clinical endpoint of MACCE at 30 days follow-up, defined as (i) all-cause mortality; (ii) non-fatal myocardial infarction; (iii) transient ischaemic attack or stroke; and (iv) heart failure with or without hospitalization (see Table 1 of Appendix for more details).

\section{Sample Size Determination}

In a recent audit of STEMI patients across several participating sub-Saharan African hospitals, we found an all-cause mortality rate of $14 \%$ and an $18 \%$ cumulative risk of readmission with post-MI heart failure requiring in-hospital treatment at 30 days. We have based our power calculations on these recent audit data, using a primary combined event rate of $30 \%$. We have estimated the effect size to be a $25 \%$ relative risk reduction in the 30-day event rate of all-cause death and heart failure hospitalization. We selected a $25 \%$ reduction as this effect size would be clinically meaningful and achievable for such a high-risk population. To demonstrate a $25 \%$ reduction in the primary composite endpoint, we will need to recruit 1078 STEMI patients in total to achieve $80 \%$ power, at the $5 \%$ significance level. Therefore, allowing for a conservative $10 \%$ drop-out rate at 30 days, we intend to recruit 1200 STEMI patients across the 20 recruiting centres over the 24 -month recruitment period. The sample size does not include participants recruited into the observational arm of the study.

\section{Statistical Analysis}

All efficacy endpoints will be analysed according to the intention-to-treat principle. The primary analysis will be a comparison of all-cause death and post-MI heart failure hospitalization event rates at 30 days after randomization between the RIC and sham control arms in all randomized STEMI participants. Differences in means among the randomized groups will be analysed using the analysis of variance (ANOVA) test and, if required, the Turkey post hoc test, and differences in proportions using the $\chi$ test, or Fisher exact test, as appropriate. Follow-up will be measured from date of randomization until date of occurrence of the composite event (death or heart failure for primary analysis, and death, non-fatal MI, transient ischaemic attack or stroke, or HF with or without hospitalization for the secondary analysis) or end of the study. The primary outcomes will also be analysed in prespecified subgroups defined in our statistical analysis plan. Survival curves of study groups will be compared using the Kaplan-Meier method, and significance will be assessed using the Log-rank test. Cox proportional hazards regression models will be fitted to identify factors associated with the hazard of occurrence of the composite event. Strength of association will be expressed as a hazard ratio, with $95 \%$ confidence intervals and Wald test $p$ value. All tests will be two-sided, and a $p$ value $<0.05$ will be considered significant. The Stata (TX, USA) statistical software will be used for data analysis.

\section{Clinical Study Monitoring and Data Management}

The Mayosi Collaborative Clinical Trials Unit at the University of Cape Town, South Africa, and the Hatter Cardiovascular Institute at the University College London, UK, will oversee the trial.

\section{Discussion}

In the RIC-AFRICA study, we intend to investigate the role of RIC as a cardioprotective strategy in patients presenting with STEMI in sub-Saharan Africa. RIC, an endogenous phenomenon achieved by using a blood pressure cuff that is simply inflated and deflated for four 5-min cycles, activates many neuro-hormonal prosurvival signalling pathways that mediate cardioprotection and preservation of left ventricular function in many small and large animal studies [19-22]. Although the actual mechanisms through which RIC exerts myocardial protection from a remotely conditioned arm remains unclear, there are a number of small proof-of-concept clinical studies that have reported benefits of RIC improving myocardial salvage after acute myocardial infarction [8, 23]. Unfortunately, the translation of RIC into clinical practice has been faced with many challenges and obstacles with negative benefits in myocardial ischaemia/refusion injury post-cardiac surgery [24, 25]. In the recent large CONDI-2/ERIC-PPCI trial, RIC was unable to improve clinical outcomes in low-risk, uncomplicated STEMI patients recruited in high-income countries that were optimally treated by PPCI [11]. The CONDI-2/ERICPPCI trial recruited mainly a low-risk patient population as evidenced by the following: (i) the low cardiac mortality rate 
of $2.7 \%$ at 12 months; (ii) $96 \%$ of the cohort having presented without symptoms or signs of heart failure (Killip class I); (iii) the median acute MI size assessed by cardiac MRI in the first week following PPCI was relatively small, with a median MI size of $17 \%$ of left ventricular mass; (iv) the total acute myocardial ischaemia time was short with a median symptom onset to PPCI time of only $3 \mathrm{~h}$; and finally, (v) the prevalence of cardiovascular risk factors were relatively low with $40 \%$ of patients having a history of hypertension, and only $10 \%$ having medically treated diabetes [13]. The benefit of RIC in higherrisk MI patients with greater comorbidities, larger infarct sizes, and longer total ischaemic times remains unknown.

The RIC-AFRICA study is particularly timely as the burden and mortality rates following STEMI experienced in lowand middle-income countries in sub-Saharan Africa are on the rise despite a decline noted globally [2,3]. Other factors contributing to the observed worse outcomes in STEMI patients in sub-Saharan Africa include the following: (i) inadequate access to hospital facilities, especially in rural areas, resulting in prolonged transfer times to facilities where thrombolytic treatment is available [26]; (ii) the increased prevalence of cardiovascular risk factors such as hypertension (in up to $60 \%$ of patients) and diabetes (in up to $40 \%$ of patients), which remains undiagnosed and untreated in many people [26]; (iii) sub-optimal use and compliance of secondary preventative guideline-directed medical therapy at discharge post-STEMI; and (iv) high total acute myocardial ischaemia times with delayed presentations ( $>6 \mathrm{~h}$ of chest pain onset) in nearly $70 \%$ of patients, confirming a higher-risk population [1].
Preliminary evidence on the safety, feasibility, and potential cardioprotective efficacy of RIC in STEMI patients treated by thrombolysis have already been demonstrated in the previously published phase 2, multi-centre, randomized controlled ERIC-LYSIS trial in the Island of Mauritius in which RIC initiated prior to thrombolysis reduced MI size as measured by a $32 \%$ reduction in 24-h area-under-the-curve serum cardiac biomarkers [23]. However, as recently discussed [12], there remains a need for a prospectively designed, randomized, sham-controlled clinical trial to determine whether RIC can improve clinical outcomes in highrisk patient groups.

Despite RIC failing to improve clinical outcomes in lowrisk STEMI patients undergoing modern reperfusion therapy with primary PCI [11], the cardioprotective efficacy of RIC in higher-risk STEMI patients treated by thrombolysis in low-and middle-income countries where PPCI is not widely available remains largely unknown and potentially an attractive, more efficacious, low-cost, and non-invasive therapeutic solution.

In summary, the RIC-AFRICA trial will determine whether the simple, low-cost, non-invasive intervention of RIC can improve clinical outcomes in higher-risk STEMI patients treated by thrombolysis.

\section{Appendix}

Table 1. Definition of study endpoints according to the 2017 cardiovascular and stroke endpoint definitions for clinical trials [17]

Pre-discharge heart failure
I. New or worsening symptoms of heart failure (dyspnoea, decreased exercise tolerance, fatigue, or
symptoms of worsened end-organ perfusion or volume overload)
II. Objective clinical evidence of heart failure and fluid retention (such as pulmonary crepitations/
crackles, raised JVP, S3 gallop, peripheral oedema, increasing abdominal distension/ ascites, or rapid
weight gain thought to be related to fluid retention)
III. Radiological evidence of pulmonary congestion/ oedema
IV. Increased cardiac biomarkers (such as BNP $>500 \mathrm{pg} / \mathrm{mL}$ or NT-proBNP $>2,000 \mathrm{pg} / \mathrm{mL}$ )
V. The initiation of intravenous or the significant augmentation of oral loop diuretic therapy
I. An admission $\geq 24 \mathrm{~h}$
II. A diagnosis of heart failure made by the treating clinician
III. The initiation of intravenous or the significant augmentation of oral loop diuretic therapy
Acute re-infarction MI that occurs in the first 28 days after the index admission for STEMI, defined by
[18]:
I. Recurrent ischaemic symptoms
II. New ST segment deviation in at least two contiguous leads
III. A $\geq 20 \%$ increase between an immediate cTn value taken at re-admission and a subsequent cTn
value taken $3-6 \mathrm{~h}$ later, exceeding the 99 th percentile upper range limit
A transient episode of focal neurological dysfunction caused by brain, spinal cord or retinal ischemia,
without acute infarction
An acute episode of focal or global neurological dysfunction caused by brain, spinal cord, or retinal
vascular injury as a result of haemorrhage or infarction
An event that meets the clinical criteria for heart failure but does not meet the length-of-hospital stay,
i.e. an admission < $24 \mathrm{~h}$


Acknowledgement This study will be undertaken in the memory of Veronica Francis, our clinical trials manager, who was instrumental in coordinating this study with all our sites in Africa. Sadly, Veronica passed away from COVID-19 in January this year, but her hard work and dedication to research will continue throughout this study.

Author Contribution All authors have read and approved the final manuscript.

Funding This study will be funded by the Mancherje-Potash Foundation in USA, and by the Hatter Cardiovascular Institute in UCL, UK. Derek Hausenloy is supported by the Duke-NUS Signature Research Programme funded by the Ministry of Health, Singapore Ministry of Health's National Medical Research Council under its Clinician Scientist-Senior Investigator scheme (NMRC/CSA-SI/0011/2017), Centre Grant, and Collaborative Centre Grant scheme (NMRC/CGAug16C006). This article is based upon work from COST Action EU-CARDIOPROTECTION CA16225 supported by COST (European Cooperation in Science and Technology)

Data Availability As this is an announcement paper, no original data has been generated.

\section{Declarations}

Ethics Approval This study was performed in line with the principles of the Declaration of Helsinki. Approval was granted by the Human Research Ethics Committee of the Faculty of Health Science, University of Cape Town (HREC107/2021).

Consent to Participate The authors affirm that informed consent will be obtained from all individual participants included in this study. Consent forms have been approved by the Human Research Ethics Committee, University of Cape Town, South Africa. As practised in most modernday STEMI trials, the RIC-AFRICA trial will offer an option for deferred consent for patients assessed to have impaired decision-making capacity. The study investigators, after providing adequate information about the trial, will assess the patient's capacity to comprehend study-related information and provide written consent. Those found to be incompetent to sign consent, a next-of-kin designated by the patient will be telephonically contacted to perform the role of proxy and provide verbal witnessed informed consent prior to randomization, which will be complemented by written consent as soon as the patient regains full capacity to do so. Clinically stable, pain-free participants deemed capable of providing written consent will consent at STEMI presentation. All participants who speak local languages other than English will have access to certified translated information leaflets and consent forms. Patients who are confused, incoherent, complicated with pulmonary oedema, or found to be haemodynamically unstable will not be recruited into the RICAFRICA trial. Patients will be informed that participation is voluntary and that they can withdraw from the study at any time, without prejudice.

Consent for Publication The authors affirm that all data collected, analysed, and published will be free from any participant identifying details. In addition, consent for the publication of anonymised data will be obtained from all individual participants included in this study before study recruitment. This study will comply with the Data Sharing Policy, and as such, this trial was registered with ClinicalTrials.gov (identifier: NCT04813159). All attempts will be made to publish the results in peer-reviewed journals. The Trial Steering Committee is responsible for reporting and publishing the results of the study. The results of the study will be submitted for publication regardless of whether the study outcomes are achieved.

Competing Interests The authors declare no competing interests.
Open Access This article is licensed under a Creative Commons Attribution 4.0 International License, which permits use, sharing, adaptation, distribution and reproduction in any medium or format, as long as you give appropriate credit to the original author(s) and the source, provide a link to the Creative Commons licence, and indicate if changes were made. The images or other third party material in this article are included in the article's Creative Commons licence, unless indicated otherwise in a credit line to the material. If material is not included in the article's Creative Commons licence and your intended use is not permitted by statutory regulation or exceeds the permitted use, you will need to obtain permission directly from the copyright holder. To view a copy of this licence, visit http://creativecommons.org/licenses/by/4.0/.

\section{References}

1. Varwani MH, Jeilan M, Ngunga M, Barasa A. Outcomes in patients with acute coronary syndrome in a referral hospital in sub-Saharan Africa. Cardiovasc J Afr. 2019;30(1):29-33.

2. Onen CL. Epidemiology of ischaemic heart disease in sub-Saharan Africa. Cardiovasc J Afr. 2013;24(2):34-42.

3. N'Guetta R, Yao H, Ekou A, N'Cho-Mottoh MP, Angoran I, Tano $\mathrm{M}$, et al. Prevalence and characteristics of acute coronary syndromes in a sub-Saharan Africa population. Ann Cardiol Angeiol (Paris). 2016;65(2):59-63.

4. Heusch G, Botker HE, Przyklenk K, Redington A, Yellon D. Remote ischemic conditioning. J Am Coll Cardiol. 2015;65(2):177-95.

5. Chong J, Bulluck H, Yap EP, Ho AF, Boisvert WA, Hausenloy DJ. Remote ischemic conditioning in ST elevation myocardial infarction - an update. Cond Med. 2018;1(5):13-22.

6. Bøtker HE, Kharbanda R, Schmidt MR, Bøttcher M, Kaltoft AK, Terkelsen CJ, et al. Remote ischaemic conditioning before hospital admission, as a complement to angioplasty, and effect on myocardial salvage in patients with acute myocardial infarction: a randomised trial. Lancet. 2010;375(9716):727-34.

7. Crimi G, Pica S, Raineri C, Bramucci E, De Ferrari GM, Klersy C, et al. Remote ischemic post-conditioning of the lower limb during primary percutaneous coronary intervention safely reduces enzymatic infarct size in anterior myocardial infarction: a randomized controlled trial. JACC Cardiovasc Interv. 2013;6(10):1055-63.

8. White SK, Frohlich GM, Sado DM, Maestrini V, Fontana M, Treibel TA, et al. Remote ischemic conditioning reduces myocardial infarct size and edema in patients with ST-segment elevation myocardial infarction. JACC Cardiovasc Interv. 2015;8(1 Pt B):178-88.

9. Sloth AD, Schmidt MR, Munk K, Kharbanda RK, Redington AN, Schmidt M, et al. Improved long-term clinical outcomes in patients with ST-elevation myocardial infarction undergoing remote ischaemic conditioning as an adjunct to primary percutaneous coronary intervention. Eur Heart J. 2014;35(3):168-75.

10. Gaspar A, Lourenco AP, Pereira MA, Azevedo P, Roncon-Albuquerque R Jr, Marques J, et al. Randomized controlled trial of remote ischaemic conditioning in ST-elevation myocardial infarction as adjuvant to primary angioplasty (RIC-STEMI). Basic Res Cardiol. 2018;113(3):14.

11. Hausenloy DJ, Kharbanda RK, Møller UK, Ramlall M, Aarøe J, Butler R, et al. Effect of remote ischaemic conditioning on clinical outcomes in patients with acute myocardial infarction (CONDI-2/ ERIC-PPCI): a single-blind randomised controlled trial. Lancet. 2019;394(10207):1415-24

12. Hausenloy DJ, Ntsekhe M, Yellon DM. A future for remote ischaemic conditioning in high-risk patients. Basic Res Cardiol. 2020;115(3):35.

13. Hausenloy DJ, Botker HE. Why did remote ischaemic conditioning not improve clinical outcomes in acute myocardial 
infarction in the CONDI-2/ERIC-PPCI trial? Cardiovasc Res. 2019;115(14):e161-e3.

14. Heusch G, Gersh BJ. Is cardioprotection salvageable? Circulation. 2020;141(6):415-7.

15. Schulz KF, Altman DG, Moher D, Group C. CONSORT 2010 statement: updated guidelines for reporting parallel group randomised trials. BMJ. 2010;340:c332.

16. Vahdatpour C, Collins D, Goldberg S. Cardiogenic Shock. J Am Heart Assoc. 2019;8(8):e011991.

17. Hicks KA, Mahaffey KW, Mehran R, Nissen SE, Wiviott SD, Dunn B, et al. 2017 Cardiovascular and stroke endpoint definitions for clinical trials. Circulation. 2018;137(9):961-72.

18. Thygesen K, Alpert JS, Jaffe AS, Chaitman BR, Bax JJ, Morrow DA, et al. Fourth universal definition of myocardial infarction (2018). Eur Heart J. 2019;40(3):237-69.

19. Hausenloy DJ, Yellon DM. Ischaemic conditioning and reperfusion injury. Nat Rev Cardiol. 2016;13(4):193-209.

20. Hausenloy DJ, Yellon DM. Remote ischaemic preconditioning: underlying mechanisms and clinical application. Cardiovasc Res. 2008;79(3):377-86.

21. Gomez L, Chavanis N, Argaud L, Chalabreysse L, Gateau-Roesch $\mathrm{O}$, Ninet $\mathrm{J}$, et al. Fas-independent mitochondrial damage triggers cardiomyocyte death after ischemia-reperfusion. Am J Physiol Heart Circ Physiol. 2005;289(5):H2153-8.
22. Santos-Gallego CG, Vahl TP, Goliasch G, Picatoste B, Arias T, Ishikawa K, et al. Sphingosine-1-phosphate receptor agonist fingolimod increases myocardial salvage and decreases adverse postinfarction left ventricular remodeling in a porcine model of ischemia/reperfusion. Circulation. 2016;133(10):954-66.

23. Yellon DM, Ackbarkhan AK, Balgobin V, Bulluck H, Deelchand A, Dhuny MR, et al. Remote ischemic conditioning reduces myocardial infarct size in STEMI patients treated by thrombolysis. J Am Coll Cardiol. 2015;65(25):2764-5.

24. Meybohm P, Bein B, Brosteanu O, Cremer J, Gruenewald M, Stoppe C, et al. A multicenter trial of remote ischemic preconditioning for heart surgery. N Engl J Med. 2015;373(15):1397-407.

25. Hausenloy DJ, Candilio L, Evans R, Ariti C, Jenkins DP, Kolvekar $\mathrm{S}$, et al. Remote ischemic preconditioning and outcomes of cardiac surgery. N Engl J Med. 2015;373(15):1408-17.

26. Bahiru E, Temu T, Gitura B, Farquhar C, Huffman MD, Bukachi F. Presentation, management and outcomes of acute coronary syndrome: a registry study from Kenyatta National Hospital in Nairobi, Kenya. Cardiovasc J Afr. 2018;29(4):225-30.

Publisher's Note Springer Nature remains neutral with regard to jurisdictional claims in published maps and institutional affiliations.

\section{Authors and Affiliations}

Kishal Lukhna ${ }^{1}$ - Derek J. Hausenloy ${ }^{2,3,4,5,6}$. Abdelbagi Sidahmed Ali ${ }^{7}$. Abdullah Bajaber ${ }^{8}$. Alistair Calver ${ }^{9}$. Arthur Mutyaba $^{10}$. Awad Abdalla Mohamed ${ }^{11,12} \cdot$ Brian Kiggundu $^{13}$. Chishala Chishala ${ }^{14}$. Ebrahim Variava ${ }^{9}$. Ehab Ali Elmakki ${ }^{15}$. Elijah Ogola ${ }^{16}$. Eltayeb Hamid ${ }^{11} \cdot$ Emmy Okello $^{13} \cdot$ Isam Gaafar $^{17} \cdot$ Keiran Mwazo $^{18}$. Makoali Makotoko ${ }^{19}$. Mergan Naidoo ${ }^{20}$. Mohamed Elhadi Abdelhameed ${ }^{21}$. Motasim Badri ${ }^{22}$. Nasief van der Schyff ${ }^{23} \cdot$ Omaima Abozaid $^{24} \cdot$ Paul Xafis $^{23} \cdot$ Sara Giesz $^{2} \cdot$ Trevor Gould $^{25} \cdot$ Waldo Welgemoed $^{25}$. Malcolm Walker ${ }^{2} \cdot$ Mpiko Ntsekhe $^{1} \cdot$ Derek M Yellon $^{2}$ (I)

1 Division of Cardiology, Groote Schuur Hospital and Faculty of Health Sciences, University of Cape Town, Cape Town, South Africa

2 The Hatter Cardiovascular Institute, University College London, London, UK

3 Cardiovascular \& Metabolic Disorders Program, Duke-National University of Singapore Medical School, Singapore, Singapore

4 National Heart Research Institute Singapore, National Heart Centre, Singapore, Singapore

5 Yong Loo Lin School of Medicine, National University Singapore, Singapore, Singapore

6 Cardiovascular Research Center, College of Medical and Health Sciences, Asia University, Taichung, Taiwan

7 Sudan Heart Centre, Khartoum, Sudan

8 Mombasa hospital, Mombasa, Kenya

9 Tshepong Hospital, Klerksdorp, South Africa

10 Division of Cardiology, Charlotte Maxeke Johannesburg Academic Hospital and University of Witwatersrand, Johannesburg, Gauteng, South Africa

11 Al Shaab Teaching Hospital, Khartoum, Sudan

12 Royal Care International Hospital, Khartoum, Sudan
13 Uganda Heart Institute, Kampala, Uganda

14 Division of Cardiology, Greys Hospital and University of KwaZulu Natal, Pietermaritzburg, South Africa

15 Aliaa Specialist Hospital, Omdurman, Sudan

16 Kenyatta National Hospital, Nairobi, Kenya

17 Omdurman Accident and Emergency Hospital, Khartoum, Sudan

18 Coast General Teaching Hospital, Mombasa, Kenya

19 Division of Cardiology, Universitas Academic Hospital, Bloemfontein, South Africa

20 Division of Family Medicine, Wentworth Hospital, University of KwaZulu Natal, Durban, South Africa

21 Al Saha Specialised Hospital, Khartoum, Sudan

22 Department of Epidemiology and Biostatistics, King Saud Bin Abdulaziz University for Health Sciences, University of Riyadh, Riyadh, Saudi Arabia

23 Victoria Hospital, University of Cape Town, Cape Town, South Africa

24 Medani Heart Centre, Wad Medani, Sudan

25 Department of Medicine, George Hospital, George, South Africa 